\title{
Influence of biological agents on cardiovascular disease in rheumatoid arthritis
}

\author{
Johan Askling, ${ }^{1,2}$ Will Dixon ${ }^{3}$
}

A wealth of studies has demonstrated that patients with rheumatoid arthritis (RA) are at increased risk of cardiovascular morbidity and mortality, and that this increase is manifest early in the course of RA. ${ }^{2}{ }^{2}$ Another wealth of studies has shown the increasing efficacy of recent therapies, or therapeutic strategies, in reducing disease activity in RA. As the inflammatory burden in RA may partly explain the increased cardiovascular risk, can effective therapy reduce the cardiovascular risk in RA? Unfortunately, and as indicated in the interesting report by Greenberg et ${ }^{\beta}{ }^{\beta}$ in this issue of the journal (pp 576-582), 'Despite the published evidence of a heightened cardiovascular risk for patients with RA, few studies have investigated therapeutic strategies to reduce this risk.' Since cardiovascular disease makes up a considerable part of the excess burden of disease and premature mortality in RA, the clinical importance of this issue cannot be underestimated. We must therefore carefully appraise the methods and results of such studies and consider future directions.

With increasing emphasis on long-term safety and disease outcomes beyond short-term EULAR DAS28 response or ACR70 we need, and are likely to see more of, studies from disease registers such as that by Greenberg et al from the CORRONA registry (reviewed in Curtis et $a l^{4}$ and Zink et al). ${ }^{5}$ However, as multiple registers address the same scientific question, we need to understand why results from different studies on the same topic may come out quite differently. Cardiovascular events following

\footnotetext{
${ }^{1}$ Clinical Epidemiology Unit, Department of Medicine Solna, Karolinska Institutet, Stockholm, Sweden ${ }^{2}$ Department of Rheumatology, Karolinska University Hospital, Stockholm, Sweden

${ }^{3}$ Arthritis Research UK Epidemiology Unit, Manchester Academic Health Sciences Centre, University of Manchester, Manchester, UK
}

Correspondence to $\mathrm{Dr}$ Johan Askling, Clinical Epidemiology Unit T2:01, Department of Medicine, Karolinska University Hospital Solna, SE-171 76 Stockholm, Sweden; Johan.askling@ki.se antitumour necrosis factor (TNF) therapy is an excellent example: the CORRONA study in this issue reports a greater than twofold reduction in cardiovascular risk for anti-TNF-treated patients compared with non-biological non-methotrexate disease-modifying antirheumatic drug (DMARD)-treated patients a finding contrasting with other null studies of anti-TNF. $^{6-9}$ They also failed to find a protective effect with methotrexate, which is somewhat at odds with previous reports. ${ }^{10}$

Transparency and harmonised reporting are key elements in this process. ${ }^{11}$ Greenberg et a $\beta$ should be commended for the reporting of their study, and do not leave the reader in doubt of the population under study, the definitions of exposure, the outcomes used, nor of their statistical approaches. Through a series of sensitivity analyses, they have challenged their own data by modifying definitions or analyses.

In essence, Greenberg et $a^{\beta}$ have compared the occurrence of cardiovascular events in three groups of patients with RA: one on anti-TNF therapy; one on methotrexate; and one on non-biological non-methotrexate DMARD therapy (the latter was used as a reference). The main result is a decreased risk of cardiovascular events with anti-TNF therapy (RR 0.39, $95 \%$ CI 0.19 to 0.82 ; ie, a $61 \%$ reduction in cardiovascular risk with anti-TNF therapy, and 95\% chance that the true effect is beyond $-18 \%$ ) but not with methotrexate (RR $0.94,95 \%$ CI 0.49 to 1.80). They also report a dose-response association between current glucocorticoid use and cardiovascular risk.

Thanks to the detailed reporting, we learn that the dramatic effect with anti-TNF therapy was not particularly sensitive to various alternative definitions of the outcome or the risk window. With this knowledge, we must first ask whether the observed results reflect a true cardiovascular risk reduction attributable to anti-TNF therapy. Second, we should consider whether differences in study design can explain the apparently discrepant findings in the literature so far. Third, we must consider how to resolve any resultant unanswered questions.

Does the dramatic reduction in relative risk associated with anti-TNF therapy really reflect a true reduction in the net risk of cardiovascular events in the CORRONA registry, or does the contrast in risk between anti-TNF therapy and non-biologicalnon-methotrexate therapy merely reflect an internal shift in the risk of cardiovascular events between different patient subsets in the CORRONA registry, ie, channeling/selection bias? As many as $43 \%$ of the study population in CORRONA were prescribed anti-TNF therapy. This high penetration (from a European perspective) raises the question of whether treatment selection is actually to or away from anti-TNF therapy in this cohort. Could the dramatically lower incidence of cardiovascular events in the anti-TNF group, evident within the first year of follow-up, be due to a high cardiovascular incidence among those patients who for some reason are not put on (or no longer receive) antiTNF therapy compared with those who are selected for (and remain on) anti-TNF therapy? As many as $21 \%$ of the nonbiological non-methotrexate DMARD comparator, one third of the methotrexate group, but only $13 \%$ of the anti-TNF group, had a history of anti-TNF therapy at the start of follow-up. Unfortunately, the sensitivity analyses that excluded all patients with a history of anti-TNF use before the start of follow-up (RR for anti-TNF $0.57,95 \%$ CI 0.22 to 1.48 ) also excluded $25 \%$ of the events, so it is not clear whether the apparent loss of effect was due to loss of precision or loss of effect, or both. Non-biological nonmethotrexate DMARD-treated patients more often had previous cardiovascular disease at baseline compared with antiTNF patients-but this was adjusted for in the analysis. Whether the accumulated burden of inflammation, which has been proposed to be a main driver of cardiovascular risk in RA, was similar or different across the three groups is not known. In order to address the issue of channeling bias within the cohort, an external cardiovascular rate might be of value. If anti-TNF therapy truly reduces cardiovascular risk, then one would expect to find that the net cardiovascular risk in the entire CORRONA registry compared with the general population was declining or non-elevated. Conversely, if the observed contrast 
between anti-TNF therapy and DMARD is not due to a lowered cardiovascular risk with anti-TNF therapy as much as a high cardiovascular risk in patients who are put on or switched to nonbiological non-methotrexate DMARD, then the overall risk across all patients in CORRONA would remain elevated compared with the general population. A similar argument could also be made in other RA cohorts and calls for the inclusion of general population reference data in future publications.

Replication of these results in other RA populations is clearly warranted with the same transparent reporting as that of Greenberg et $a l^{3}$ as is a better understanding of why previous observational studies have not found the same result. Greenberg et $a{ }^{\beta}$ list some methodological differences, but a more exhaustive comparison (and more new data) could perhaps tell us whether these differences are mainly related to differences in 'quality' or internal validity (ie, the likelihood that the study result is a true answer to the question posed) or whether the various study populations used harbour important differences. Their methods, results and appropriate sensitivity analyses exclude several sources of bias, although the authors correctly acknowledge possible residual confounding (eg, for glucocorticoids, which are intimately associated with disease activity/severity, or a difficulty in finding a better therapy for the RA disease, both of which may in turn be associated with cardiovascular risk). Estimation of some drug discontinuation dates as the midpoint between visits may have underestimated the incidence in anti-TNF-treated patients, potentially introducing bias. The issue of generalisability or external validity is particularly important as it could indicate that any protective effect is restricted to patients with specific characteristics. The identification of such characteristics could help move us towards personalised medicine in this era of increasing choice of biological agents.

Observational studies provide an excellent setting to address questions of drug safety, despite the challenges of accurate interpretation. The authors correctly state that 'trials of TNF antagonists have not been designed to study cardiovascular endpoints...', and most reasonably chose to perform a large observational study. Whereas their other purported reason for not using a trial meta-analysis ("... have insufficient power to detect modest benefits in relatively uncommon outcomes, such as MI.') certainly held true in the light of findings from previous studies on cardiovascular risks with anti-TNF therapy, their intriguing finding of a greater than $50 \%$ reduction in cardiovascular events detectable within 1 year of anti-TNF therapy suggests this is not necessarily true any longer. Although cardiovascular events such as fatal and non-fatal myocardial infarctions and strokes have not been predefined trial outcomes in the efficacy trials of antiTNF in RA, such events would qualify as adverse events in the same trials and get reported as such. There is some evidence that a meta-analysis approach would work. In a recent meta-analysis of corporate sponsored randomised controlled trials of adalimimab, etanercept and infliximab aimed at assessing the short-term risk of cancer with these therapies, 74 trials encompassing 15000 patients (9700 person-years) treated with anti-TNF therapy versus 7500 patients (4400 person-years) randomly assigned to comparator therapies were identified, the adverse events reports could be searched for potential cancer events, and the case narratives could be adjudicated. ${ }^{12}$ Assuming the incidence of the cardiovascular outcome in these trials is similar to that reported from CORRONA (2.7 vs 7.5 per 1000 personyears for anti-TNF vs non-biological non-methotrexate DMARD), one would expect the anti-TNF trial experience to generate approximately 60 events, which is comparable to the 82 events in the study by Greenberg et al. ${ }^{3}$ In our view, a reasonable next step that would circumvent some of the issues with observational designs (although generate other issues) would be to query the same trial experience for cardiovascular events as an adjudicated yet post-hoc outcome. Further down the line, a strategy trial in RA with cardiovascular events as the outcome is not unrealistic.

As evidence accumulates in this important topic, we move closer towards a true understanding of the effect of drug therapies on cardiovascular outcomes. Even apparently discrepant findings provide us with important information, although this is only possible with thoughtful study design, analysis and high-quality reporting. As things stand, it is still not clear to what extent anti-TNF therapy influences cardiovascular disease, and if so whether this is a specific effect of antiTNF therapy or a generic effect related to improved disease control in RA, whichever way it is achieved. Additional wellconducted observational studies and/or meta-analyses of clinical trials may hold the key. In the meantime, we should not forget to intervene against the usual suspects in terms of cardiovascular risk factors when we know intervention is effective.

Provenance and peer review Commissioned; externally peer reviewed.

Accepted 2 January 2011

Ann Rheum Dis 2011;70:561-562

doi:10.1136/ard.2010.142174

\section{REFERENCES}

1. Kremers HM, Crowson CS, Therneau TM, et al. High ten-year risk of cardiovascular disease in newly diagnosed rheumatoid arthritis patients: a population-based cohort study. Arthritis Rheum 2008;58:2268-74.

2. Aviña-Zubieta JA, Choi HK, Sadatsafavi M, et al. Risk of cardiovascular mortality in patients with rheumatoid arthritis: a meta-analysis of observational studies. Arthritis Rheum 2008;59:1690-7.

3. Greenberg JD, Kremer JM, Curtis JR, et al. Tumour necrosis factor antagonist use and associated risk reduction of cardiovascular events among patients with rheumatoid arthritis. Ann Rheum Dis 2011:70:576-582.

4. Curtis JR, Jain A, Askling J, et al. A comparison of patient characteristics and outcomes in selected European and U.S. rheumatoid arthritis registries. Semin Arthritis Rheum 2010;40:2-14.e1.

5. Zink A, Askling J, Dixon WG, et al. European biologicals registers: methodology, selected results and perspectives. Ann Rheum Dis 2009;68:1240-6.

6. Dixon WG, Watson KD, Lunt M, et al. Reduction in the incidence of myocardial infarction in patients with rheumatoid arthritis who respond to antitumor necrosis factor alpha therapy: results from the British Society for Rheumatology Biologics Register. Arthritis Rheum 2007:56:2905-12.

7. Solomon DH, Avorn J, Katz JN, et al. Immunosuppressive medications and hospitalization for cardiovascular events in patients with rheumatoid arthritis. Arthritis Rheum 2006;54:3790-8.

8. Suissa S, Bernatsky S, Hudson M. Antirheumatic drug use and the risk of acute myocardial infarction. Arthritis Rheum 2006;55:531-6.

9. Barnabe C, Martin BJ, Ghali WA. Systematic review and meta-analysis: anti-tumor necrosis factor alpha therapy and cardiovascular events in rheumatoid arthritis. Arthritis Care Res (Hoboken). Published Online First: 18 October 2010. PMID 20957658.

10. Choi HK, Hernán MA, Seeger JD, et al. Methotrexate and mortality in patients with rheumatoid arthritis: a prospective study. Lancet 2002;359:1173-7.

11. Dixon WG, Carmona L, Finckh A, et al. EULAR points to consider when establishing, analysing and reporting safety data of biologics registers in rheumatology. Ann Rheum Dis 2010;69:1596-602.

12. Askling J, Fahrbach $\mathrm{K}$, Nordstrom B, et al. Cancer risk with tumor necrosis factor alpha (TNF) inhibitors: meta-analysis of randomized controlled trials of adalimumab, etanercept, and infliximab using patient level data. Pharmacoepidemiol Drug Saf 2011;20:119-30. 